\title{
Transcellular biosynthesis contributes to the production of leukotrienes during inflammatory responses in vivo
}

\author{
Jean-Etienne Fabre, ${ }^{1}$ Jennifer L. Goulet, ${ }^{1}$ Estelle Riche, ${ }^{2}$ MyTrang Nguyen, ${ }^{1}$ \\ Kenneth Coggins, ${ }^{1}$ Steven Offenbacher, ${ }^{2}$ and Beverly H. Koller ${ }^{1}$ \\ ${ }^{1}$ Department of Genetics, University of North Carolina Chapel Hill, Chapel Hill, North Carolina, USA \\ ${ }^{2}$ Center for Oral and Systemic Diseases, University of North Carolina Chapel Hill, School of Dentistry, Chapel Hill, \\ North Carolina, USA \\ Address correspondence to: Beverly H. Koller, Department of Genetics, Campus Box 7248, Thurston Bowles Building, \\ University of North Carolina Chapel Hill, Chapel Hill, North Carolina 27599, USA. \\ Phone: (919) 962-2153; Fax: (919) 966-7524; E-mail: Treawouns@aol.com.
}

Received for publication December 17, 2001, and accepted in revised form April 10, 2002.

\begin{abstract}
Leukotrienes are lipid mediators that evoke primarily proinflammatory responses by activating receptors present on virtually all cells. The production of leukotrienes is tightly regulated, and expression of 5-lipoxygenase, the enzyme required for the first step in leukotriene synthesis, is generally restricted to leukocytes. Arachidonic acid released from the cell membrane of activated leukocytes is rapidly converted to LTA 4 by 5-lipoxygenase. $\mathrm{LTA}_{4}$ is further metabolized to either $\mathrm{LTC}_{4} \mathrm{or} \mathrm{LTB}_{4}$ by the enzyme $\mathrm{LTC}_{4}$ synthase or $\mathrm{LTA}_{4}$ hydrolase, respectively. Unlike 5-lipoxygenase, these enzymes are expressed in most tissues. This observation previously has led to the suggestion that LTA $\mathrm{L}_{4}$ produced by leukocytes may, in some cases, be delivered to other cell types before being converted into $\mathrm{LTC}_{4}$ or $\mathrm{LTB}_{4}$. While in vitro studies indicate that this process, termed transcellular biosynthesis, can lead to the production of leukotrienes, it has not been possible to determine the significance of this pathway in vivo. Using a series of bone marrow chimeras generated from 5-lipoxygenase- and $\mathrm{LTA}_{4}$ hydrolase-deficient mice, we show here that transcellular biosynthesis contributes to the production of leukotrienes in vivo and that leukotrienes produced by this pathway are sufficient to contribute significantly to the physiological changes that characterize an ongoing inflammatory response.
\end{abstract}

J. Clin. Invest. 109:1373-1380 (2002). doi:10.1172/JCI200214869.

\section{Introduction}

Leukotrienes (LTs) belong to a large family of lipid mediators, termed eicosanoids, that are derived from arachidonic acid (AA) and released from the cell membrane by phospholipases. They can be found at high levels in most inflammatory lesions and have been shown to contribute to the physiological changes observed during inflammatory responses. The first step in the production of all LTs, the oxygenation of AA to form 5-hydroperoxyeicosatretranoic acid and the immediate dehydration of this unstable intermediate to $\mathrm{LT} \mathrm{A}_{4}\left(\mathrm{LTA}_{4}\right)$, is carried out by 5 -lipoxygenase (5-LO). $\mathrm{LTA}_{4}$ produced from AA by 5-LO is the common substrate from which all LTs are formed. Metabolism of $\mathrm{LTA}_{4}$ by $\mathrm{LTA}_{4}$ hydrolase $\left(\mathrm{LTA}_{4}-\mathrm{H}\right)$ results in the production of the potent chemoattractant $\mathrm{LT} \mathrm{B}_{4}$ $\left(\mathrm{LTB}_{4}\right)$. Alternatively, $\mathrm{LTA}_{4}$ can be conjugated with glutathione by $\mathrm{LTC}_{4}$ synthase $\left(\mathrm{LTC}_{4}-\mathrm{S}\right)$ to produce $\mathrm{LT} \mathrm{C}_{4}$ $\left(\mathrm{LTC}_{4}\right)$ and its metabolites $\mathrm{LTD}_{4}$ and $\mathrm{E}_{4}$, collectively referred to as the cysteinyl LTs. These lipid mediators are known to increase vascular permeability and regulate smooth muscle tone.

The enzyme required for the initiation of production of all LTs, 5-LO, is expressed predominantly by cells of myeloid origin, particularly neutrophils, eosinophils, monocytes/macrophages, and mast cells
(1-4). In contrast, the enzymes carrying out the second step in LT biosynthesis, $\mathrm{LTC}_{4}-\mathrm{S}$ and $\mathrm{LTA}_{4}-\mathrm{H}$, are more broadly expressed, and high levels of $\mathrm{LTA}_{4}-\mathrm{H}$ expression have been noted in several tissues $(4,5)$. These findings have suggested that under some circumstances $\mathrm{LTA}_{4}$ produced by inflammatory cells can be transferred to cells that express only $\mathrm{LTA}_{4}-\mathrm{H}$, where the synthesis of $\mathrm{LTB}_{4}$ is then completed.

Evidence suggesting that individual steps in the production of eicosanoids might occur in different cells comes, in part, from experiments showing that plateletderived arachidonate may be used by endothelial cells to produce prostacyclin (6). The process was termed "transcellular metabolism" or "transcellular biosynthesis." A number of lines of evidence support a role for transcellular biosynthesis in the production of LTs. First, labeling studies showed that, similar to the production of prostacyclin, AA present in platelets could be used by neutrophils to produce $\mathrm{LTB}_{4}$ (7). Thus, AA used in the production of LTs can be provided by neighboring cells and does not need to come only from the activated myeloid cells. Furthermore, experiments showing that $\mathrm{LTA}_{4}$ is released by activated polymorphonuclear leukocytes suggested that not only AA, but also the metabolic intermediate $\mathrm{LTA}_{4}$, might be transferred between cells (8-10). Finally, results from a 
number of coculture systems using purified populations of cells, tissues, and organs, are consistent with a model in which at least a portion of LTs present in tissue are produced by transcellular biosynthesis. In these studies, the level and profile of the LTs produced from individual cell populations were examined and compared with those produced when two populations were cultured together. These studies showed that both the amount and type of LTs present in the supernatant from the combined populations often differed from that expected by simple addition of the LTs produced by the individually cultured cells. For example, while neutrophils stimulated with the ionophore A23187 produce primarily $\mathrm{LTB}_{4}$ and isolated lung tissue produces only very low levels of $\mathrm{LTC}_{4}$, coculture of lung tissue with neutrophils resulted in a marked increase in $\mathrm{LTC}_{4}$ production. Similar results were obtained from coculture of neutrophils with either erythrocytes (11, 12), keratinocytes (13), alveolar macrophages (14), tracheal cells (15), platelets (16-18), or endothelial cells (19), or with (20) isolated organs, such as skin (13), lung $(21,22)$, and heart (23).

Despite this intense investigation, it has not been possible to determine whether transcellular biosynthesis occurs in vivo and, if so, whether the levels of eicosanoids produced by this pathway are sufficient to contribute to the ongoing inflammatory response. To examine this question directly we use two genetically engineered mouse lines; the first is deficient in 5- $\mathrm{LO}\left(5-\mathrm{Lo}^{-/-}\right)$, and the second is deficient in $\mathrm{LTA}_{4}-\mathrm{H}$ $\left.\left(\operatorname{Lta}_{4}{ }^{-b^{-/}}\right)^{-}\right)$. We and others have shown previously $(24$, 25) that the 5- $\mathrm{Lo}^{-/-}$mice fail to produce both $\mathrm{LTB}_{4}$ and $\mathrm{LTC}_{4}$ and thus have blunted inflammatory responses to peritoneal injection of zymosan A and to cutaneous application of AA (26). As expected, the $\mathrm{Lta}_{4} \mathrm{~b}^{-/}$ animals are deficient in production of $\mathrm{LTB}_{4}$, while biosynthesis of $\mathrm{LTC}_{4}$ remains intact and is not decreased (27). The response of these mice to peritoneal injection of zymosan A and to cutaneous application of AA is also attenuated (27). Using cells from these two mouse lines and bone marrow chimeras generated from these animals, we show that transcellular metabolism can occur in vivo and that the LTs produced may, in at least some instances, contribute to an inflammatory response.

\section{Methods}

Mice. The generation of 5- $\mathrm{LO}^{-/-}$and $\mathrm{Lta}_{4} \mathrm{~b}^{-/-}$mice has been described previously $(26,27)$. These mutant alleles are maintained on a 129 genetic background of $129 /$ Ola and $129 / \mathrm{SvEv}$ origin. All mice studied were at least 8 weeks old. All experiments were approved by the Institutional Animal Care and Use Committee.

Induction of $L T B_{4}$ synthesis by freshly isolated peritoneal lenkocytes. Prior to the induction of peritonitis with zymosan A, animals were treated with a single intravenous injection (10 mg/kg body weight) of indomethacin (Sigma-Aldrich, St. Louis, Missouri, USA) to inhibit prostaglandin production.
Zymosan A (Sigma-Aldrich) was suspended at $55^{\circ} \mathrm{C}$ in sterile PBS to a final concentration of $1 \mathrm{mg} / \mathrm{ml}$, and $1 \mathrm{ml}$ was injected into the peritoneal cavity. Three hours later, mice were killed and the peritoneal cavity lavaged with $4 \mathrm{ml}$ of PBS. Lavage fluid (2 $\mathrm{ml} /$ mouse) was collected in polypropylene tubes on ice, and cells were collected by centrifugation. Cell pellets were washed in HBSS and resuspended in HBSS at $10^{7} \mathrm{cells} / \mathrm{ml}$. Six hundred microliters of each cell suspension was transferred to a 12 -well plate. After 5 minutes' incubation at $37^{\circ} \mathrm{C}$, cells were treated with A23187 (Sigma-Aldrich) for 30 minutes at $37^{\circ} \mathrm{C}$. The media was then collected and frozen for later measurement of $\mathrm{LTB}_{4}$ levels.

$L T B_{4}$ detection. Proteins present in the media or lavage fluid to be analyzed $(500 \mu \mathrm{l})$ were precipitated by addition of cold ethanol $(2 \mathrm{ml})$, followed by centrifugation at $15,000 \mathrm{~g}$ at $4^{\circ} \mathrm{C}$ for 10 minutes. The supernatant was acidified with $8 \mathrm{ml}$ of $0.1 \mathrm{M}$ phosphate buffer, $\mathrm{pH} 3.5$, and passed through an activated C18 column (Sep-Pak Plus; Waters Corporation, Milford, Massachusetts, USA). The column was washed twice and then eluted with ethanol/water (90:10, vol/vol). The eluate was dried under nitrogen and resuspended in ELISA buffer. $\mathrm{LTB}_{4}$ levels were determined by ELISA (Cayman Chemical, Ann Arbor, Michigan, USA).

Generation of bone marrow chimeras. Groups of six female recipient mice were placed in a ventilated container and exposed to 9 Gy at $80 \mathrm{cGy} / \mathrm{min}$ from a cobalt source. Within 3 hours of irradiation of female mice, male donors were killed and bone marrow cell suspensions were prepared from femurs by repeated flushing with $2 \mathrm{ml}$ of PBS. Bone marrow cell suspensions were filtered through Miracloth (22- to $25-\mu \mathrm{m}$ pores; Calbiochem-Novabiochem Corp., La Jolla, California, USA). Each female recipient received, by intravenous tail vein injection, the whole bone marrow cells harvested from one male, resuspended in $0.5 \mathrm{ml}$ of saline. Transplanted mice were fed normal mouse chow without antibiotic supplementation. To verify that successful bone marrow reconstitution had occurred, mice were anesthetized, and $100 \mu \mathrm{l}$ of blood was drawn from the orbital sinus and mixed with 700 $\mu \mathrm{l}$ of saline containing $1 \mathrm{mM}$ EDTA. After centrifugation, cell pellets were resuspended in a specific buffer for lysis of red blood cells and centrifuged again. This process was repeated three times. The final pellet was resuspended in PCR buffer with proteinase $\mathrm{K}$ and incubated at $55^{\circ} \mathrm{C}$ for 1 hour. Proteinase $\mathrm{K}$ was inactivated at $95^{\circ} \mathrm{C}$ for 10 minutes, and $3 \mu$ of this preparation (about $1 \mu \mathrm{g}$ DNA) was analyzed for the presence of the Y chromosome by PCR using the following primers designed to detect the Sry gene: $5^{\prime}$-TGTTCAGCCCTACAGCCACATG- $3^{\prime}$ and 5'-CCACTCCTCTGTGACACTTTAGCC- $3^{\prime}$ (30 seconds at $94^{\circ} \mathrm{C}, 30$ seconds at $56^{\circ} \mathrm{C}$, and 90 seconds at $72^{\circ} \mathrm{C}$ for 30 cycles). Under these conditions, a 340-bp fragment is amplified when the blood sample contains male cells. 


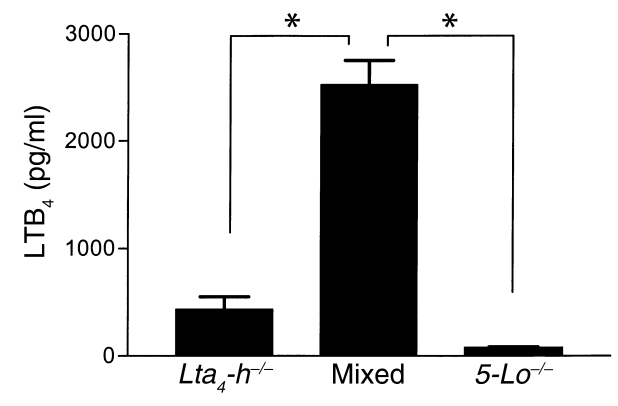

Figure 1

In vitro $\mathrm{LTB}_{4}$ production by leukocytes stimulated with A23187. Leukocytes were isolated from peritoneal lavage fluid of mice in which acute peritonitis had been induced by injection of zymosan $A$. The 5- $\mathrm{Lo}^{-/-}$and $\mathrm{Lta}_{4} \mathrm{~h}^{-/-}$leukocytes do not produce significant amounts of $\mathrm{LTB}_{4}$ in response to stimulation with $\mathrm{A} 23187$. In contrast, similar treatment of a mixture of these two cell populations resulted in a significant increase in $\mathrm{LTB}_{4}$ production. This graph shows data averaged from three separate experiments. ${ }^{*} P<0.01$.

Zymosan A-induced peritoneal inflammation. Peritonitis was induced with zymosan A as described above, except the peritoneal cavity was lavaged 3 hours later. The lavage samples were centrifuged at $12,000 \mathrm{~g}$ at $4^{\circ} \mathrm{C}$ for 10 minutes and the supernatant stored at $-80^{\circ} \mathrm{C}$ until analysis.

AA induced cutaneous inflammation. Animals were injected intravenously with indomethacin $(10 \mathrm{mg} / \mathrm{kg}$ body weight) and $0.5 \%$ Evans blue dye $(10 \mathrm{ml}$ of dye solution per kilogram of body weight). Evans blue dye (Sigma-Aldrich) was dissolved in PBS at $\mathrm{pH}$ 7.5. The inner surface of the pinna of the left ear of each mouse was painted with $20 \mu \mathrm{l}$ of AA $(100 \mu \mathrm{g} / \mu \mathrm{l}$ in acetone), while the right ear was treated with an equal amount of acetone alone. One hour after AA treatment, mice were sacrificed, and an 8-mm-diameter disc of tissue was punched from the center of each ear and weighed. To extract the Evans blue dye, ear biopsies were incubated in $1 \mathrm{ml}$ of formamide at $55^{\circ} \mathrm{C}$ for 48 hours. Serum protein extravasation was quantified by measuring the absorbance of the formamide extracts at $610 \mathrm{~nm}\left(\mathrm{~A}_{610}\right)$.

Statistical analysis. Data are presented as the mean plus or minus SEM. Log-rank test for survival analysis and one-way ANOVA with Newman-Keul's post test for inflammatory responses analysis were performed using GraphPad Prism version 3.00 for Windows (GraphPad Software for Science Inc., San Diego, California, USA). A $P$ value of less than 0.05 was interpreted to denote statistical significance.

\section{Results}

In vitro evidence for transcellular biosynthesis using 5- $\mathrm{Lo}^{-/-}$ or $\mathrm{Lta}_{4} \mathrm{-b}^{-/-}$cells. To determine whether mice deficient in different enzymes required for the production of $\mathrm{LTB}_{4}$ can be used to study the contribution of transcellular metabolism to the production of LTs in vivo, we tested whether leukocytes isolated from these mouse lines can cooperate in the production of $\mathrm{LTB}_{4}$ in an in vitro assay. To obtain mouse leukocytes, we elicited an inflammatory response by peritoneal injection of zymosan A. Three hours after injection of zymosan A, greater than $90 \%$ of the cells present in the peritoneal lavage from these mice are neutrophils. Cells were treated with the ionophore A23187 to stimulate the release of AA from the cell membrane by phospholipase $\mathrm{A}_{2}$ and the subsequent metabolism of this substrate into LTs. As expected, no or barely detectable amounts of $\mathrm{LTB}_{4}$ were present in the medium after treatment of either $5-\mathrm{Lo}^{-/-}$or $\mathrm{Lta}_{4} \mathrm{~b}^{-/-}$leukocytes with A23187 (Figure 1). In contrast, a significant increase in the $\mathrm{LTB}_{4}$ level was measured in medium prepared from identically treated samples that consisted of a mixture of equal numbers of cells from 5- $\mathrm{Lo}^{-/-}$and $\mathrm{Lta}_{4} \mathrm{~h}^{-\mathrm{H}^{-}}$mice. The results of this experiment are consistent with a model in which the synthesis of LTs that is initiated in one cell can be completed in neighboring cells after the transfer of intermediate metabolites.

Generation of chimeric mouse lines. To determine whether $\mathrm{LTA}_{4}$ produced by 5 -LO in one cell can be metabolized to $\mathrm{LTB}_{4}$ by the $\mathrm{LTA}_{4}-\mathrm{H}$ expressed in a different cell in vivo, we used a model in which 5-LO-deficient mice are irradiated and transplanted with bone marrow prepared from wild-type mice or mice deficient in either 5-LO or $\mathrm{LTA}_{4}-\mathrm{H}$ (Figure 2). The following bone marrow chimera populations were generated from $5-\mathrm{Lo}^{-/-}, \mathrm{Lta}_{4} \mathrm{~b}^{-/-}$, and wild-type mice. First, we generated a population of mice to control for the possible impact of irradiation

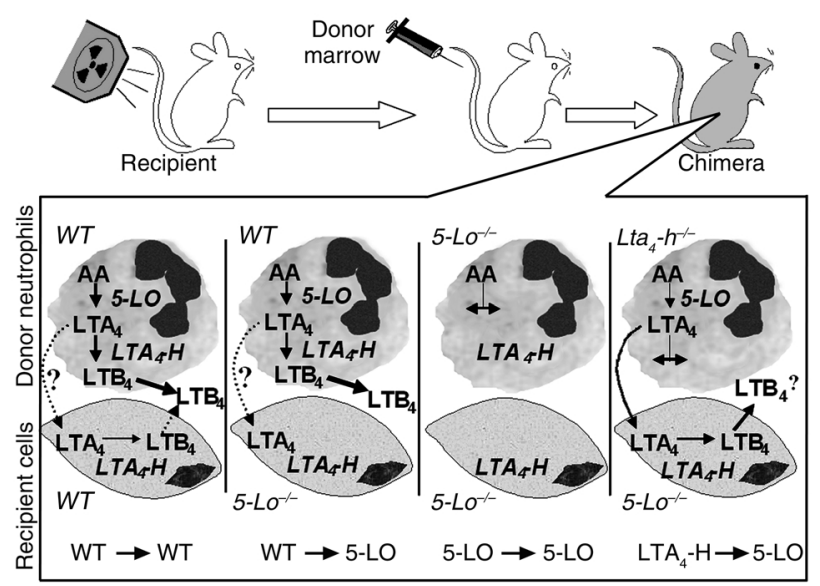

Figure 2

Description of the experiments. Chimeric mice are generated by exposing mice to a lethal dose of ionizing radiation with subsequent injection of donor bone marrow. The following chimeras were generated: wild-type mice receiving wild-type marrow $(\mathrm{WT} \rightarrow \mathrm{WT})$, $5-\mathrm{Lo}^{-/-}$mice receiving wild-type marrow $(\mathrm{WT} \rightarrow 5-\mathrm{LO}), 5-\mathrm{Lo}^{-/-}$mice receiving $5-\mathrm{Lo}^{-/-}$marrow $(5-\mathrm{LO} \rightarrow 5-\mathrm{LO})$, and $5-\mathrm{Lo}^{-/-}$mice receiving $\mathrm{Lta}_{4} \mathrm{~h}^{-/-}$marrow $\left(\mathrm{LTA}_{4}-\mathrm{H} \rightarrow 5-\mathrm{LO}\right)$. The callout illustration shows the expected interaction between neutrophils of donor origin (top) and the cells of recipient origin (bottom) that will lead to $\mathrm{LTB}_{4}$ production. Labels in the illustration refer to the donors and to the recipients, respectively (donor genotype $\rightarrow$ recipient genotype). WT, wildtype mice; $\mathrm{LTA}_{4}-\mathrm{H}$, $\mathrm{LTA}_{4}-\mathrm{H}$-deficient mice; 5-LO, 5-LO-deficient mice; $A A$, arachidonic acid. 
$\mathbf{a}$
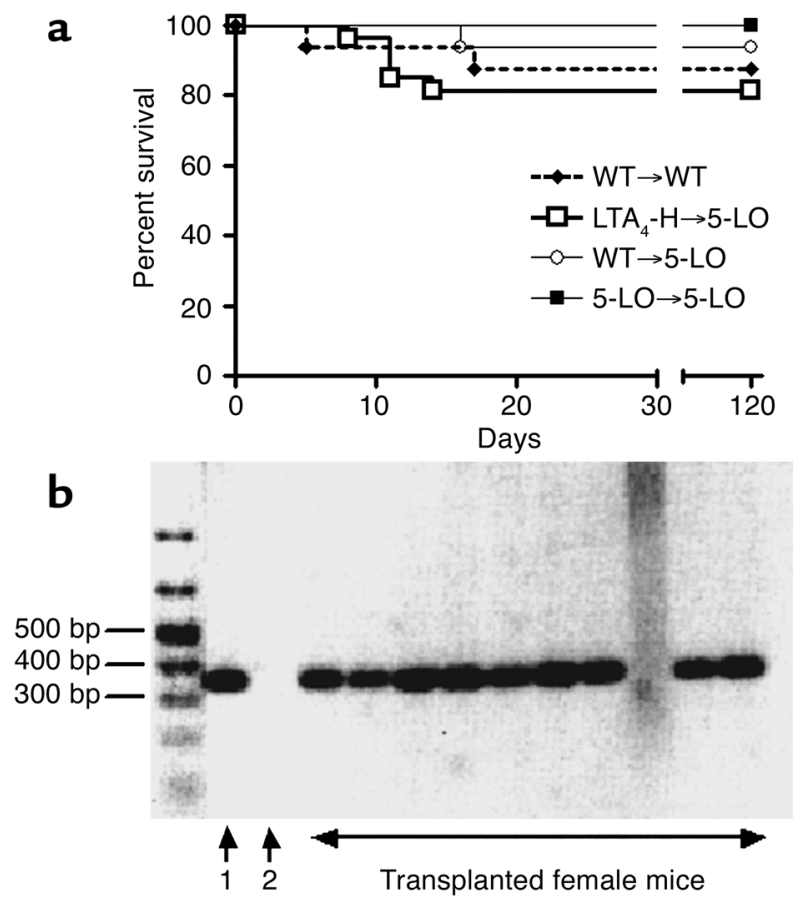

treatment on the mice and/or changes in hematopoietic cell populations in reconstituted animals on the inflammatory response of the mice. This population consisted of wild-type 129 mice reconstituted with bone marrow cells isolated from other wild-type animals $(\mathrm{WT} \rightarrow \mathrm{WT})$. A second control population was generated by irradiation of 5- $\mathrm{Lo}^{-/-}$mice followed by reconstitution with $5-\mathrm{Lo}^{-/-}$bone marrow $(5-\mathrm{LO} \rightarrow 5-\mathrm{LO})$. This population served as a control for possible changes in the

\section{Figure 4}

Transcellular biosynthesis in vivo. (a) Zymosan A-induced peritoneal inflammation. $\mathrm{LTB}_{4}$ production was measured in the peritoneal lavage fluid. Loss of 5-LO expression impairs $\mathrm{LTB}_{4}$ production in response to zymosan A stimulation (5-LO $\rightarrow 5-\mathrm{LO})$. In contrast, the presence of leukocytes expressing 5-LO, but unable to produce $\mathrm{LTB}_{4}\left(\mathrm{LTA}_{4}-\mathrm{H} \rightarrow 5-\mathrm{LO}\right)$, results in $\mathrm{LTB}_{4}$ production. ${ }^{*} P<0.05$. WT, wild-type; 5-LO, 5-LO-deficient mice; $\mathrm{LTA}_{4}-\mathrm{H}$, $\mathrm{LTA}_{4}-\mathrm{H}$-deficient mice. The transplanted groups are identified as donor $\rightarrow$ recipient genotypes. Results are presented as numerical values in Table 1. (b) Acute cutaneous inflammatory responses to AA. Mice received Evans blue dye and indomethacin immediately before topical application of AA. At 1 hour after the treatment, the inflamed tissue was biopsied, weighed, and the serum exudate present in the tissue was determined. The white bars represent the difference in optical densities ( $\triangle \mathrm{OD}$ ) between the ear treated with $\mathrm{AA}$ and the ear treated with vehicle (acetone) alone. The black bars represent the difference in ear-disc weights ( $\Delta$ ear weight) between the AA-treated ear and the vehicle-treated ear for each mouse. While loss of 5-LO or $\mathrm{LTA}_{4}-\mathrm{H}$ attenuated the inflammatory response to $A A$, the response was significantly increased in mice in which the reconstituted immune cells express 5-LO, but are unable to produce $\mathrm{LTB}_{4}\left(\mathrm{LTA}_{4}-\mathrm{H} \rightarrow 5 \mathrm{LO}\right)$. This graph combines data from two separate experiments. Statistical significance is indicated only for the transplanted groups for clarity. ${ }^{*} P<0.05$. WT, wild-type; 5-LO, 5-LO-deficient mice; $\mathrm{LTA}_{4}-\mathrm{H}$, $\mathrm{LTA}_{4}-\mathrm{H}$-deficient mice. Results are presented as numerical values in Table 1.

\section{Figure 3}

Efficiency of bone marrow transplantation. (a) Survival curve obtained after irradiation and reconstitution with marrow from various genotypes. Symbols refer to the donors and to the recipients, respectively (donor genotype $\rightarrow$ recipient genotype). WT, wild-type mice; $\mathrm{LTA}_{4}-\mathrm{H}$, LTA 4 - $\mathrm{H}$-deficient mice; 5-LO, 5-LO-deficient mice. (b) PCR detection of the $Y$ chromosome in total DNA extracted from blood of female mice transplanted with marrow from male donors. Lane 1 shows expression of the $Y$ chromosome as a 340-bp band detected in blood from a male control. Lane 2 was loaded with DNA from a nontransplanted female. Other lanes were loaded with DNA from transplanted females.

dependence of various inflammatory responses on LTs after the irradiation and reconstitution treatment protocol in the deficient mice. Two experimental populations of bone marrow chimeras were generated. One group was generated by irradiation of 5-LO-deficient mice and reconstitution of these mice with wild-type bone marrow (WT $\rightarrow 5-\mathrm{LO}$ ). These animals allowed us to measure the contribution of LTs derived from hematopoietic cells to the inflammatory response. For the other experimental group, we irradiated $5-\mathrm{Lo}^{-/-}$mice and reconstituted these animals with bone marrow from $\mathrm{Lta}_{4} \mathrm{~h}^{-/-}$animals $\left(\mathrm{LTA}_{4}-\mathrm{H} \rightarrow 5-\mathrm{LO}\right)$. We expected that the resulting chimeras would have myeloid populations capable of producing $\mathrm{LTA}_{4}$, but that, because of the lack of $\mathrm{LTA}_{4}-\mathrm{H}$, no $\mathrm{LTB}_{4}$ could be produced without cooperation between these cells and cells of the recipient animal (Figure 2a, right panel).

Wild-type mice $(n=11)$ exposed to 9 Gy of ionizing irradiation died 8-15 days after treatment (data not shown). When the mice received bone marrow

a
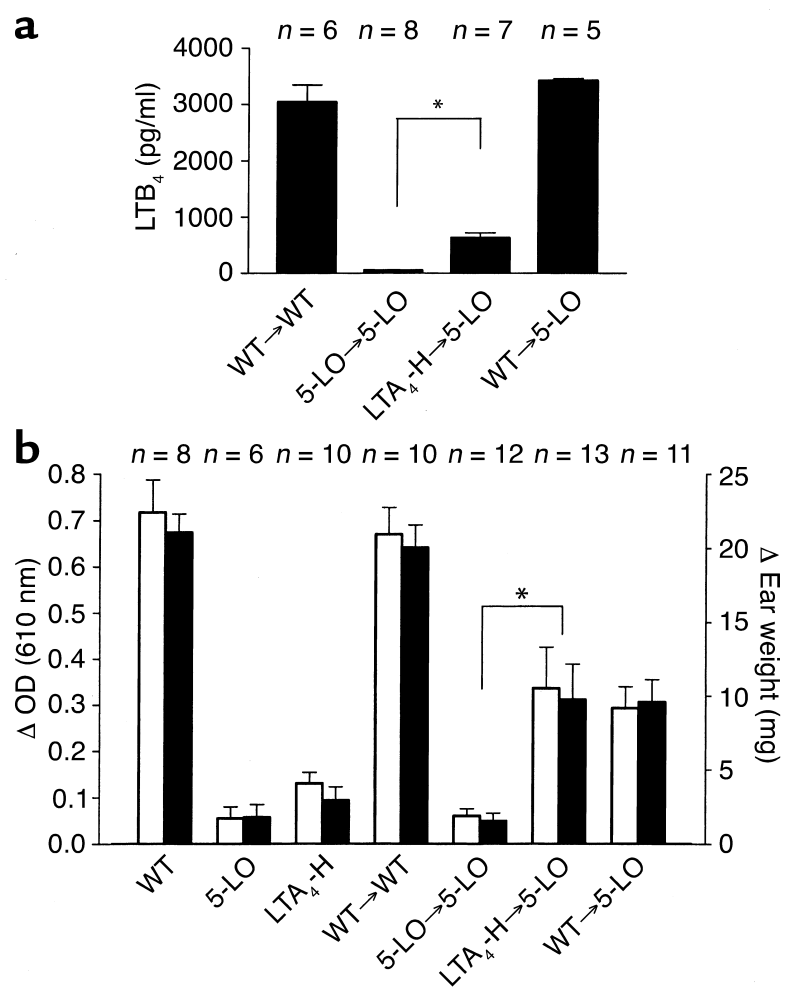
Table 1

Numerical data obtained from experiments demonstrating the transcellular biosynthesis in vivo

\begin{tabular}{lccc}
\hline & $\begin{array}{c}\text { Ear } \Delta \text { weight } \\
(\text { gram })\end{array}$ & $\begin{array}{c}\text { Ear } \Delta \text { OD } \\
\text { (absorbance units) }\end{array}$ & $\begin{array}{c}\text { Peritoneal LTB } \\
\text { (picogram/milliliter) }\end{array}$ \\
WT & $21.1 \pm 1.23(n=9)$ & $0.717 \pm 0.070(n=8)$ & \\
$5-\mathrm{LO}$ & $1.81 \pm 0.87(n=7)$ & $0.055 \pm 0.025(n=6)$ & \\
$\mathrm{LTA}_{4}$-H & $2.93 \pm 0.93(n=10)$ & $0.131 \pm 0.024(n=10)$ & \\
WT/WT & $20.0 \pm 1.53(n=10)$ & $0.670 \pm 0.058(n=10)$ & $3,040 \pm 299(n=6)$ \\
$5-\mathrm{LO} / 5-\mathrm{LO}$ & $1.54 \pm 0.54(n=12)$ & $0.060 \pm 0.015(n=12)$ & $51.5 \pm 11(n=8)$ \\
$\mathrm{LTA}_{4}$-H/5-LO & $9.25 \pm 2.41(n=13)$ & $0.336 \pm 0.089(n=13)$ & $630 \pm 88(n=7)$ \\
WT/5-LO & $9.59 \pm 1.53(n=11)$ & $0.294 \pm 0.046(n=11)$ & $3,420 \pm 38(n=5)$ \\
\hline
\end{tabular}

which are able to generate $\mathrm{LTA}_{4}$ but not convert it to $\mathrm{LTB}_{4}$, encountered 5-LO-deficient but $\mathrm{LTA}_{4}-\mathrm{H}$-sufficient recipient cells in their vicinity.

Cutaneous inflammatory response to $\mathrm{AA}$ in $\mathrm{Lta}_{4} \mathrm{~h}^{-/-} / 5-\mathrm{LO}^{-/-}$bone marrow chimeras. We next sought to determine whether transcellular metabolism could produce sufficient $\mathrm{LTB}_{4}$ to significantly contribute to the physiological changes observed during an inflammatory response. We

transplants within 6 hours after exposure to the irradiation, most (80\%) of the animals survived and remained healthy for over 3 months (Figure 3a); although there was a slightly lower survival of the $\mathrm{LTA}_{4}-\mathrm{H} \rightarrow 5$-LO population, this difference did not reach statistical significance $(P>0.05)$. This finding is consistent with the successful engraftment of the bone marrow and reconstitution of the immune system and was true for all chimeras generated from wild-type, 5- $\mathrm{Lo}^{-/-}$, and $\mathrm{Lta}_{4}{ }^{-b^{-/-}}$mice (log-rank test: $P=0.26$ ). Because the bone marrow used for transplantation was prepared from male mice, and in all cases the recipient animals were females, PCR amplification of DNA sequences specific to the $\mathrm{Y}$ chromosome was used to monitor reconstitution of the irradiated mice with donor hematopoietic cells, as shown in Figure $3 \mathrm{~b}$.

$\mathrm{LTB}_{4}$ production in $\mathrm{Lta}_{4} \mathrm{~h}^{-/-} / 5-\mathrm{Lo}^{-/-}$bone marrow chimeras. Zymosan A is a purified polysaccharide fraction of yeast cell wall that elicits a strong inflammatory response when introduced into the peritoneal cavity. This response is characterized by an influx of neutrophils by 2 hours, and a measurable increase in $\mathrm{LTB}_{4}$ biosynthesis at 3 hours, which mainly is due to the infiltration of the phagocytic cells. The level of $\mathrm{LTB}_{4}$ production in peritoneal lavage fluid in response to zymosan A was shown previously to be dramatically decreased in $\mathrm{Lta}_{4} \mathrm{~h}^{-/-}$mice (27). We therefore used this model to determine whether measurable levels of $\mathrm{LTB}_{4}$ could be produced by transcellular metabolism in vivo.

As shown in Figure $4 \mathrm{a}$ and Table 1, peritoneal lavage fluid obtained from wild-type mice treated with zymosan A contained high levels of $\mathrm{LTB}_{4}$. As expected, $\mathrm{LTB}_{4}$ levels were barely detectable in the lavage fluid obtained from similarly treated $5-\mathrm{LO}^{-/-}$mice reconstituted with $5-\mathrm{Lo}^{-/-}$marrow. In contrast, $\mathrm{LTB}_{4}$ production was restored in the $5-\mathrm{Lo}^{-/-}$mice reconstituted with wild-type bone marrow, supporting the hypothesis that the majority of $\mathrm{LTB}_{4}$ is produced by the neutrophilic infiltrate characteristic of this inflammatory response. The $5-\mathrm{Lo}^{-/-}$mice transplanted with $\mathrm{Lta}_{4} \mathrm{~b}^{-/-}$marrow produced more $\mathrm{LTB}_{4}$ than the 5- $\mathrm{LO}^{-/-}$mice grafted with syngenic marrow. These data suggest that $\mathrm{LTB}_{4}$ production increased significantly when donor $\mathrm{LTA}_{4}-\mathrm{H}$-deficient leukocytes, have shown previously that 5-LO- and $\mathrm{LTA}_{4}-\mathrm{H}-$ deficient mice have an attenuated inflammatory response to cutaneous application of AA (25-27). When these mice are treated with the cyclooxygenase inhibitor indomethacin to block the production of prostaglandins, both the edema formation and influx of neutrophils characteristic of the response are almost entirely dependent on LT production.

To test the ability of the different mice generated to mount an inflammatory response to topical application of AA on ear tissue, we measured both the weight of ear biopsy samples and the extravasation of plasma fluid (as represented by extravasation of Evans blue dye, which binds to serum proteins). In addition to the four groups of bone marrow chimeras, wild-type mice and 5-LO- and $\mathrm{LTA}_{4}-\mathrm{H}$-deficient mice were included in the study.

As can be seen in Figure $4 \mathrm{~b}$ and Table 1, application of AA to the pinna of the ear of wild-type 129 mice led to a dramatic change in ear weight that was accompanied by increased protein extravasation into the tissue. A response of similar magnitude was elicited in the wild-type mice reconstituted with isogenic bone marrow (compare WT with WT $\rightarrow$ WT). This finding suggests that the treatment of mice with irradiation, followed by bone marrow reconstitution, did not significantly alter the ability of mice to respond to AA. Similar to our previous results, the inflammatory response to cutaneous application of AA was significantly attenuated in the 5- $\mathrm{LO}^{-/-}$mice (compare 5-LO with WT). These data are consistent with earlier studies showing that in indomethacin-treated animals this inflammatory response is dependent on the production of LTs by the 5-LO pathway. The dependence of this response on expression of 5-LO was also observed in the 5- $\mathrm{LO}^{-/-}$mice that had been irradiated and reconstituted with syngenic bone marrow (compare 5-LO with 5 -LO $\rightarrow 5$-LO). We reported previously that edema and plasma protein extravasation after topical application of AA is reduced considerably in mice lacking $\mathrm{LTA}_{4}-\mathrm{H}$, demonstrating the $\mathrm{LTB}_{4}$ contribution to this response (27). Consistent with our previous results, the inflammatory response was significantly attenuated in mice deficient in expression of $\mathrm{LTA}_{4}-\mathrm{H}$, although not to the low levels observed in the 5-LO-deficient 
animals (compare $\mathrm{LTA}_{4}-\mathrm{H}$ with WT). The response remaining in these mice is likely due to the continued production of $\mathrm{LTC}_{4}$ by the $\mathrm{LTA}_{4}-\mathrm{H}$-deficient mice. These findings indicate that the increase in ear weight and edema formation is largely dependent on the production of $\mathrm{LTB}_{4}$ and recruitment of leukocytes to the site of inflammation. The defective response of the 5-LO-deficient mice was partially corrected by reconstitution of the hematopoietic system with bone marrow from congenic wild-type animals (compare 5 - $\mathrm{LO} \rightarrow 5$-LO with $\mathrm{WT} \rightarrow 5-\mathrm{LO}$ ). However, this response remained less vigorous than that observed in the wild-type animals. Transplantation of the 5-LO-deficient mice with bone marrow from $\mathrm{LTA}_{4}-\mathrm{H}-$ deficient mice also restored, in part, the ability of these mice to respond to AA (compare 5-LO $\rightarrow 5-\mathrm{LO}$ with $\mathrm{LTA}_{4}-\mathrm{H} \rightarrow 5-\mathrm{LO}$ ).

\section{Discussion}

Previous studies have suggested a model in which the profile and level of eicosanoids produced during an inflammatory response reflect, in part, the cooperation between myeloid and nonmyeloid cells in their biosynthesis. However, direct proof that transcellular biosynthesis of LTs occurs in vivo has been difficult to obtain. We show here the utility of using bone marrow chimeras and genetically modified animals to address this question. While neither $\mathrm{Lta}_{4} \mathrm{~b}^{-/-}$nor $5-\mathrm{Lo}^{-/-}$animals produce $\mathrm{LTB}_{4}$, this $\mathrm{LT}$ can be detected in bone marrow chimeras generated from these two mouse lines. Furthermore, we show that $\mathrm{LTB}_{4}$ produced by transcellular biosynthesis can restore the ability of the mice to mount an inflammatory response to AA.

Production of $\mathrm{LTB}_{4}$ by transcellular biosynthesis was examined after induction of peritonitis in the bone marrow chimeras with zymosan A. Zymosan A is known to elicit an inflammatory response characterized by infiltrates composed primarily of neutrophils within 3-6 hours after treatment. Previously, we showed that while zymosan $\mathrm{A}$-induced peritonitis is characterized by high levels of $\mathrm{LTB}_{4}$ in wild-type mice, $\mathrm{LTB}_{4}$ could not be detected in lavage fluid from $\mathrm{Lta}_{4}-\mathrm{h}^{-/-}$mice. Because of the high levels of $\mathrm{LTB}_{4}$ that can be measured in this response, we chose this system to determine whether, in this particular inflammatory response, $\mathrm{LTA}_{4}$ produced by neutrophils could be transferred to other cells for further metabolism into $\mathrm{LTB}_{4}$. Bone marrow chimeras were generated in which the majority of the hematopoietic cells express 5-LO but not $\mathrm{LTA}_{4}-\mathrm{H}$. Neutrophils normally do not produce significant amounts of $\mathrm{LTC}_{4}$ because of low levels of LTC $_{4}$-S. Thus, upon stimulation of the neutrophils, $\mathrm{LTA}_{4}$ is produced but is likely to accumulate in these $\mathrm{LTA}_{4}-\mathrm{H}$-deficient cells. In these bone marrow chimeras, nonmyeloid cells are unable to metabolize AA into $\mathrm{LTA}_{4}$ because they do not express 5-LO. However, these cells express $\mathrm{LTA}_{4}-\mathrm{H}$. If the $\mathrm{LTA}_{4}$ produced by the neutrophils is transferred to these cells, then we would expect to observe $\mathrm{LTB}_{4}$ in the lavage fluid. We report here that this transcellular biosynthesis does occur and can produce about $20 \%$ of the $\mathrm{LTB}_{4}$ levels observed when the reconstituted population of cells express both 5- $\mathrm{LO}$ and $\mathrm{LTA}_{4}-\mathrm{H}$.

The production of $\mathrm{LTB}_{4}$ by transcellular biosynthesis in the bone marrow chimeras, however, might be an overestimate of the contribution of this pathway to $\mathrm{LTB}_{4}$ production in wild-type mice for the following reason. LTA $_{4}$ might never accumulate in wild-type neutrophils because of its rapid conversion by $\mathrm{LTA}_{4}-\mathrm{H}$ present in the same cell. Thus, the production of $\mathrm{LTB}_{4}$ by transcellular biosynthesis we observed might be unique to this experimental system. Conversely, the amount of $\mathrm{LTB}_{4}$ produced by transcellular biosynthesis might be very dependent on the ratio of inflammatory cells to nonhematopoietic cells and the level of $\mathrm{LTA}_{4}-\mathrm{H}$ in these acceptor cells. It is possible that, in this model of peritonitis, a high ratio of inflammatory cells to parenchyma cells reduces the contribution of the transcellular metabolic pathways to the overall production of the LTs. Furthermore, we cannot rule out the possibility that some of the $\mathrm{LTB}_{4}$ we have measured in the $\mathrm{LTA}_{4}-\mathrm{H} \rightarrow 5$ - $\mathrm{LO}$ chimeras is the result of transcellular biosynthesis between neutrophils of donor and recipient origin. While our data clearly show that the donor bone marrow cells have engrafted, some survival and eventual contribution of the recipient hematopoietic cells is possible. Thus, a small percentage of the neutrophils might be 5-LO deficient and $\mathrm{LTA}_{4}-\mathrm{H}$ sufficient. These cells could contribute to the production of the observed $\mathrm{LTB}_{4}$. However, even if this were the case, our measurement of $\mathrm{LTB}_{4}$ clearly shows that transcellular metabolism can occur during an in vivo inflammatory response.

To determine whether LTs produced by transcellular biosynthesis could contribute to an inflammatory response, we used the AA-induced model of cutaneous inflammation. While AA is not a physiologically relevant inflammatory stimulus, this model has several characteristics that made it ideal for these initial in vivo studies. In this model, the contribution of LTs to the inflammatory response can be isolated by pretreatment of the mice with indomethacin, a cyclooxygenase inhibitor. After treatment with indomethacin, 5-LO-deficient mice fail to respond to AA. In addition, we have shown previously that the response to AA of mice deficient in $\mathrm{LTA}_{4}-\mathrm{H}$ is blunted (27). Furthermore, we show here that when the $\mathrm{LTA}_{4}-\mathrm{H}$-deficient mice are treated with indomethacin, edema formation and increases in ear weight in response to AA are not significantly greater than that observed in the 5-LO-deficient animals.

To carry out these experiments, it was first necessary to determine the extent to which cells of hematopoietic origin contributed to AA-induced cutaneous inflammation. This was done by comparing the inflammatory response in wild-type mice reconstituted with wild-type bone marrow (WT $\rightarrow \mathrm{WT}$ ) with the inflammatory response observed in 5-LO-deficient mice reconstituted with wild-type bone marrow 
(WT $\rightarrow 5-\mathrm{LO}$ ). We were surprised to find that plasma protein extravasation and changes in tissue weight in response to AA in mice in which the parenchyma cells were 5 -LO deficient (WT $\rightarrow 5$-LO) were only about half of that observed in wild-type mice (WT $\rightarrow$ WT). Two explanations for this observation are possible. First, cells of nonhematopoietic origin might contribute to this response. Some evidence supporting this possibility comes from in vitro culture studies in which production of LTs from keratinocytes was demonstrated (28). Alternatively, some recovery of the recipient hematopoietic cells might have occurred; therefore, at least a small portion of the hematopoietic cells is 5-LO deficient and unable to produce LTs. However, the observation that the level of $\mathrm{LTB}_{4}$ in the lavage fluid of the $\mathrm{LTA}_{4}-\mathrm{H} \rightarrow \mathrm{WT}$ and WT $\rightarrow$ WT chimera is similar (and production of this mediator has been attributed to the recruited neutrophils) suggests that the majority of the myeloid cells are of donor origin. This would be consistent with the former hypothesis, namely that some nonhematopoietic cells can produce LTs. Future experiments that monitor not only engraftment but also survival of recipient marrow should allow us to further rule out the possibility that the decreased inflammatory response observed in the 5-LO-deficient mice reconstituted with wild-type marrow can be attributed to residual 5- $\mathrm{Lo}^{-/-}$myeloid populations.

Irradiation and subsequent reconstitution of 5-LO-deficient mice with $\mathrm{LTA}_{4}-\mathrm{H}$-deficient marrow $\left(\mathrm{LTA}_{4}-\mathrm{H} \rightarrow 5-\mathrm{LO}\right)$ resulted in dramatic increases in inflammation as compared with 5 -LO-deficient mice reconstituted with syngenic marrow $(5-\mathrm{LO} \rightarrow 5$-LO). Although the synthesis of $\mathrm{LTB}_{4}$ is not specifically measured in the cutaneous model, the increased response in the $\mathrm{LTA}_{4}-\mathrm{H} \rightarrow 5-\mathrm{LO}$ group probably results from $\mathrm{LTB}_{4}$ produced by transcellular conversion of $\mathrm{LTA}_{4}$. It is unlikely that the response in these mice is due to production of $\mathrm{LTC}_{4}$ by the hematopoietic cells because the response of the $\mathrm{LTA}_{4}-\mathrm{H} \rightarrow 5$ - $\mathrm{LO}$ mice is significantly higher than that observed in the $\mathrm{LTA}_{4}-\mathrm{H}$-deficient mice. The loss of $\mathrm{LTA}_{4}-\mathrm{H}$ has a large impact on edema formation in this model despite that fact that cells deficient in this enzyme, which express 5-LO and $\mathrm{LTC}_{4}$ synthase, can still produce $\mathrm{LTC}_{4}$ and subcutaneous injection of $\mathrm{LTC}_{4}$ can itself induce edema formation. This suggests that there is significant synergy between the two classes of LTs, LTB 4 and the cysteinyl LTs, in this response. It should be possible to test this in the future with the development of mice deficient only in the production or response to $\mathrm{LTC}_{4}$. We cannot exclude the possibility that increased lipoxin production resulting from shunting into this pathway contributes to the decreased edema seen in the $\mathrm{LTA}_{4}-\mathrm{H}$-deficient mice (29). Conversely, we cannot rule out the possibility that the restoration of the response in the chimeras $\left(\mathrm{LTA}_{4} \rightarrow 5-\mathrm{LO}\right)$ is due not only to production of $\mathrm{LTB}_{4}$ by transcellular metabolism, but in part reflects alterations in lipoxin production, for example lower levels of lipoxins in the $\mathrm{LTA}_{4}-\mathrm{H} \rightarrow 5$ - $\mathrm{LO}$ versus $\mathrm{LTA}_{4}$ mice.
LTs are potent mediators of inflammation and are capable of inducing all of the cardinal signs of inflammation. $\mathrm{LTB}_{4}$ is one of the most potent chemoattractants for neutrophils, and $\mathrm{LTC}_{4}$, originally identified as the slow-reacting substance of anaphylaxis, is one of the most potent activators of contraction of airway smooth muscle identified to date and is also a potent vasoconstricting agent. It is, therefore, perhaps not surprising that the production of these lipid mediators is tightly regulated and that it is generally limited to inflammatory responses. The transcellular biosynthesis of LTs might provide an important mechanism for regulating the production of these lipid mediators during an inflammatory response. The absence of 5-LO expression in tissues protects the organism from the production of these lipids, but also limits the levels of LTs that can be produced in the early minutes of the inflammatory response. Transcellular biosynthesis might provide a means for maximizing the production of LTs from recruited myeloid cells. Excess $\mathrm{LTA}_{4}$ produced by these cells could be metabolized into active lipid mediators, not only by $\mathrm{LTA}_{4}-\mathrm{H}$ in the myeloid cell itself, but also by adjacent cells in the inflamed tissue.

The studies reported here provide direct evidence that transcellular biosynthesis contributes in vivo to the production of $\mathrm{LTB}_{4}$ in two experimental models of inflammation: zymosan A-induced peritonitis and AA-stimulated cutaneous inflammation. Future studies using bone marrow chimeras generated from 5- $\mathrm{Lo}^{-1-}, \mathrm{Lta}_{4} \mathrm{~b}^{-1-}$, and $\mathrm{Ltc}_{4} \mathrm{~S}^{-\mathrm{S}^{--}}$mice should allow the role of transcellular biosynthesis to be studied in other inflammatory responses and to be extended to the production of other LTs, such as LTC $_{4}$.

\section{Acknowledgments}

The authors thank V.A. Wagoner for assistance in animal husbandry, P. Flannery for assistance in leukotriene detection, and J. Snouwaert for reviewing the manuscript. This work was supported in part by grants from the NIH (P01 DK-38108 to B.H. Koller and NIDCR 1-P60 DE-13079 to S. Offenbacher) and by a grant from the American Heart Association (9920376U to J.-E. Fabre).

\footnotetext{
1. Miller, D.K., et al. 1990. Identification and isolation of a membrane protein necessary for leukotriene production. Nature. 343:278-281.

2. Dixon, R.A., et al. 1990 . Requirement of a 5-lipoxygenase-activating protein for leukotriene synthesis. Nature. 343:282-284.

3. Reid, G.K., et al. 1990. Correlation between expression of 5-lipoxygenaseactivating protein, 5-lipoxygenase, and cellular leukotriene synthesis. J. Biol. Chem. 265:19818-19823.

4. Samuelsson, B., and Funk, C.D. 1989. Enzymes involved in the biosynthesis of leukotriene B4. J. Biol. Chem. 264:19469-19472.

5. Radmark, O., et al. 1989. Leukotriene A4-hydrolase, cloning of the human enzyme and tissue distribution in the rat. Adv. Prostaglandin Thromboxane Leukot. Res. 19:484-487.

6. Marcus, A.J., Weksler, B.B., Jaffe, E.A., and Broekman, M.J. 1980. Synthesis of prostacyclin from platelet-derived endoperoxides by cultured human endothelial cells. J. Clin. Invest. 66:979-986.

7. Marcus, A.J., et al. 1982. Formation of leukotrienes and other hydroxy acids during platelet-neutrophil interactions in vitro. Biochem. Biophys. Res. Commun. 109:130-137.

8. Radmark, O., et al. 1980. Leukotriene A: stereochemistry and enzymatic conversion to leukotriene B. Biochem. Biophys. Res. Commun. 92:954-961.
} 
9. Dahinden, C.A., Clancy, R.M., Gross, M., Chiller, J.M., and Hugli, T.E. 1985. Leukotriene C4 production by murine mast cells: evidence of a role for extracellular leukotriene A4. Proc. Natl. Acad. Sci. USA 82:6632-6636

10. Sala, A., Bolla, M., Zarini, S., Muller-Peddinghaus, R., and Folco, G. 1996. Release of leukotriene A4 versus leukotriene B4 from human polymorphonuclear leukocytes. J. Biol. Chem. 271:17944-17948.

11. Fitzpatrick, F., et al. 1984. Metabolism of leukotriene A4 by human erythrocytes. A novel cellular source of leukotriene B4. J. Biol. Chem. 259:11403-11407.

12. McGee, J.E., and Fitzpatrick, F.A. 1986. Erythrocyte-neutrophil interactions: formation of leukotriene B4 by transcellular biosynthesis. Proc. Natl. Acad. Sci. USA. 83:1349-1353

13. Iversen, L., et al. 1994. Identification and subcellular localization of leukotriene A4-hydrolase activity in human epidermis. J. Dermatol. Sci. 7:191-201.

14. Grimminger, F., Sibelius, U., and Seeger, W. 1991. Amplification of LTB4 generation in AM-PMN cocultures: transcellular 5-lipoxygenase metabolism. Am. J. Physiol. 261:L195-L203.

15. Bigby, T.D. 1991. Transcellular metabolism of leukotrienes in the lung. Adv. Exp. Med. Biol. 314:235-250.

16. Pace-Asciak, C.R. 1985. Profiling of prostaglandins and related products by GC/MS with negative-ion chemical-ionization detection. Adv Prostaglandin Thromboxane Leukot. Res. 15:39-43.

17. Edenius, C., Haeggstrom, J., and Lindgren, J.A. 1988. Transcellular conversion of endogenous arachidonic acid to lipoxins in mixed human platelet-granulocyte suspensions [erratum 1989, 159:370]. Biochem. Biophys. Res. Commun. 157:801-807.

18. Maclouf, J.A., and Murphy, R.C. 1988. Transcellular metabolism of neutrophil-derived leukotriene A4 by human platelets. A potential cellular source of leukotriene C4. J. Biol. Chem. 263:174-181.

19. Feinmark, S.J., and Cannon, P.J. 1986. Endothelial cell leukotriene C4 synthesis results from intercellular transfer of leukotriene A4 synthesized by polymorphonuclear leukocytes. J. Biol. Chem. 261:16466-16472.

20. Claesson, H.E., and Haeggstrom, J. 1988. Human endothelial cells stim- ulate leukotriene synthesis and convert granulocyte released leukotriene A4 into leukotrienes B4, C4, D4, and E4. Eur. J. Biochem. 173:93-100.

21. Grimminger, F., Menger, M., Becker, G., and Seeger, W. 1988. Potentiation of leukotriene production following sequestration of neutrophils in isolated lungs: indirect evidence for intercellular leukotriene A4 transfer. Blood. 72:1687-1692.

22. Grimminger, F., Kreusler, B., Schneider, U., Becker, G., and Seeger, W. 1990. Influence of microvascular adherence on neutrophil leukotriene generation. Evidence for cooperative eicosanoid synthesis. J. Immunol. 144:1866-1872

23. Sala, A., et al. 2000. Monoclonal anti-CD18 antibody prevents transcellular biosynthesis of cysteinyl leukotrienes in vitro and in vivo and protects against leukotriene-dependent increase in coronary vascular resistance and myocardial stiffness. Circulation. 101:1436-1440.

24. Funk, C.D., Chen, X.S., Kurre, U., and Griffis, G. 1995. Leukotriene-deficient mice generated by targeted disruption of the 5-lipoxygenase gene. Adv. Prostaglandin Thromboxane Leukot. Res. 23:145-150.

25. Byrum, R.S., Goulet, J.L., Griffiths, R.J., and Koller, B.H. 1997. Role of the 5 -lipoxygenase protein (FLAP) in murine acute inflammatory responses. J. Exp. Med. 6:1065-1075.

26. Goulet, J.L., Snouwaert, J.N., Latour, A.M., Coffman, T.M., and Koller, B.H. 1994. Altered inflammatory responses in leukotriene-deficient mice. Proc. Natl. Acad. Sci. USA. 91:12852-12856.

27. Byrum, R.S., Goulet, J.L., Snouwaert, J.N., Griffiths, R.J., and Koller, B.H. 1999. Determination of the contribution of cysteinyl leukotrienes and leukotriene B4 in acute inflammatory responses using 5-lipoxygenaseand leukotriene A4 hydrolase-deficient mice. J. Immunol. 163:6810-6819.

28. Janssen-Timmen, U., et al. 1995. 5-lipoxygenase expression in cultured human keratinocytes. Adv. Prostaglandin Thromboxane Leukot. Res. 23:329-331.

29. Serhan, C.N., Takano, T., Chiang, N., Gronert, K., and Clish, CB. 2000 Formation of endogenous "antiinflammatory" lipid mediators by transcellular biosynthesis. Lipoxins and aspirin-triggered lipoxins inhibit neutrophil recruitment and vascular permeability. Am. J. Respir. Crit. Care Med. 161(Suppl.):S95-S101. 